\title{
Vibration isolation stacks for gravitational wave detectors-Finite element analysis
}

\author{
C. A. Cantley, J. Hough, and N. A. Robertson \\ Department of Physics \& Astronomy, University of Glasgow, Glasgow G12 8QQ, Scotland \\ R. J. S. Greenhalgh \\ Rutherford Appleton Laboratory, Chilton, Didcot, Oxon OX11 0QX, England
}

(Received 11 October 1991; accepted for publication 2 January 1992)

\begin{abstract}
Seismic isolation is a necessity for many delicate experiments such as those designed to search for gravitational radiation. One method of providing a significant amount of isolation has been the use of multiple stage stacks of alternating layers of dense material (metal) and elastic material (rubber). In this work finite element analysis is used to model stack systems in a relatively realistic way, allowing the importance of cross coupling of degrees of freedom to be evaluated. It becomes clear that care has to be taken with the geometrical construction in order to achieve the isolation predicted by simple dynamical analysis.
\end{abstract}

\section{INTRODUCTION}

Gravitational wave detectors currently being developed rely on sensing the extremely small motions which are expected to be produced by a gravitational wave passing through a mechanical system. The system may be a bar of low loss metal cooled to liquid-helium temperatures' ${ }^{1}$ or a series of test masses which are hung as pendulums at the ends of two perpendicular arms of a laser interferometer. ${ }^{2}$ In both of these cases it is essential to isolate the mechanical system from the surroundings to reduce spurious noise introduced by local seismic or mechanical disturbances. In general the detector systems are arranged so that the motions to be monitored lie in the horizontal plane. Thus it is particularly important that the vibration isolation system is designed such that it can provide a high degree of horizontal isolation. However, since geometrical effects can crosscouple motion in one direction into motion in another it is sensible to aim for isolation factors in the remaining degrees of freedom (vertical, tilt, and rotation) which are suitably large. In most designs of gravitational wave detector, pendulum suspensions are used to provide a significant degree of isolation but in general further isolation is required. This may be provided by placing vibration isolation stacks, consisting of alternating layers of metal and elastic material, between the pendulum suspension points and the ground.

Using simple dynamical theory the transmissibility of unidirectional motion at the base of a vibration isolation stack to the corresponding displacement at the center of mass of the top plate of the stack can be readily estimated as a function of frequency. In such an analysis the stack is treated as a system of point masses connected with springs and dampers. In practice however, cross coupling is liable to be an important factor in determining the effectiveness of the isolation stack. It is not straightforward to develop an analytical model which includes the effects of distributed masses-and it is precisely these effects which can lead to cross coupling. One of our principal objectives in this work has been to quantify the degree of cross coupling occurring within a particular design of isolation stack. For this purpose a finite element model representing an aluminum plate supported by four rubber pieces was generated to represent one stage of a stack and the finite element program MSC/NASTRAN, ${ }^{3,4}$ was used to carry out various analyses. The model dimensions are shown in Fig. 1 and the resulting resonant frequencies are typical of what could be applicable to a prototype laser interferometric gravitational wave detector. Note that to achieve the best overall isolation with such a system the ratio of the rubber stiffness to the mass of the supported plate is chosen to give the system a reasonably low resonant frequency in each dimension.

Investigations were conducted on a "single stage" stack model and also on a "double stage" which consisted of two single stages in series. The effects of a stiffness imbalance of the rubber at either end of a given stage of stack were also investigated. The results of these tests were generalized to allow an understanding of the performance of a stack containing four stages. Up to five stages have been considered in the planning of the seismic isolation systems for long baseline detectors. ${ }^{2}$

The work discussed in this article is most relevant to gravitational wave detectors which use laser interferometry to sense relative motions of separated masses suspended as pendulums, but is also of relevance in any field where stacks may be used as part or all of an isolation system.

\section{PERFORMANCE REQUIRED FROM VIBRATION ISOLATION STACKS}

The performance required from a stack system depends on the particular application, but we assume that the performance needed is such that an interferometric gravitational wave detector of arm length $3 \mathrm{~km}$ may approach a sensitivity for measuring strains in space of $\sim 10^{-24}$, $\sqrt{\mathrm{Hz}}$ at $100 \mathrm{~Hz}^{2}$ We also assume that the test masses are suspended in the simplest way, by wires as simple pendulums with horizontal and vertical resonant frequencies of 1 and $20 \mathrm{~Hz}$, respectively. In this case, at $100 \mathrm{~Hz}$, move- 


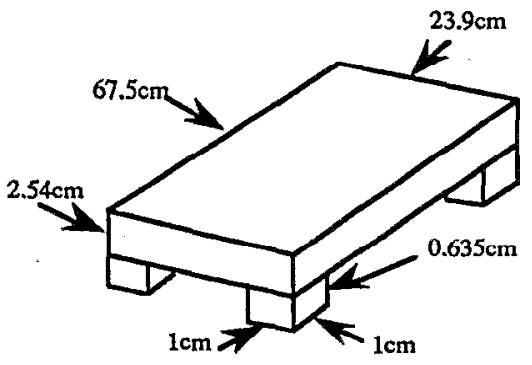

'TWO-DIMENSIONAL' REPRESENTATION

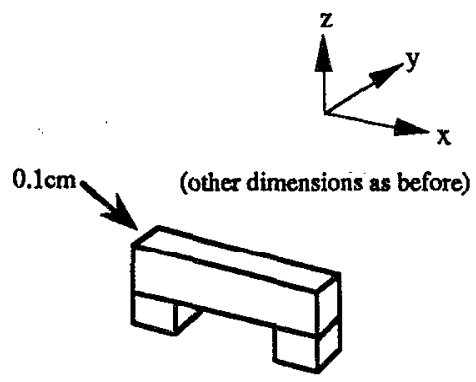

FIG. 1. Dimensions of the two-dimensional finite element model compared to the three-dimensional stack. ments at the top of the stack of less than $4 \times 10^{-18} \mathrm{~m} /$ $\sqrt{\mathrm{Hz}}$ in the vertical direction and $1.5 \times 10^{-17} \mathrm{~m} / \sqrt{\mathrm{Hz}}$ in the horizontal direction are required. A figure of $1 \%$ coupling of vertical into horizontal movement in the pendulum suspension has been used to calculate the vertical limit as such a figure has been suggested by the experimental measurements of Del Fabbro et al. ${ }^{5}$

Obviously the isolation required from the stack depends on the magnitude of ground movement in the appropriate sense. A figure of $\sim 10^{-11} \mathrm{~m} / \sqrt{\mathrm{Hz}}$ at $100 \mathrm{~Hz}$, with a spectrum falling at $12 \mathrm{~dB}$ per octave, was assumed in the horizontal and vertical directions for the work in this paper; ${ }^{2}$ and ground tilt of $\sim 2 \times 10^{-11} \mathrm{rad} / \sqrt{\mathrm{Hz}}$ at $100 \mathrm{~Hz}$, as recently measured at a prototype detector site, with a spectrum falling somewhere between $12 \mathrm{~dB}$ per octave and $18 \mathrm{~dB}$ per octave, was used. ${ }^{6}$

\section{GENERATION OF THE FINITE ELEMENT MODEL}

It was decided to represent the three-dimensional system with what was essentially a two-dimensional crosssectional slice through it in the $x-z$ plane (see Fig. 1). This was done so that the complexity involved in the generation of the model and in the interpretation of the results obtained was kept to a minimum. Note that in modeling the stack in this way the investigations involving rotational motion were restricted to those where the axes of rotation were parallel to the $y$ axis (tilting motions).

The structure was modeled by generating a mesh of nodes and connecting them with plate elements having the desired physical properties (see Fig. 2). Note that some of the physical properties of the materials used in the model had to be scaled so that the two-dimensional finite element model exhibited behavior appropriate to its three-dimensional counterpart.

Synthetic rubber is extensively used in isolation stacks. The horizontal and vertical resonant frequencies $\left(f_{h}\right.$ and $f_{u}$ ) of the plate (mass $m=11.1 \mathrm{Kg}$ ) on four pieces of Neoprene rubber of the size shown in Fig. 1 were deter-

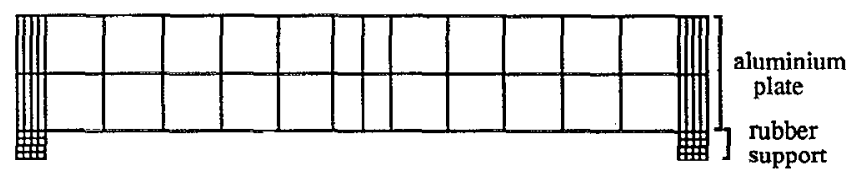

FIG. 2. Finite element model of the one-stage vibration isolation stack. The system is divided into a number of elements as shown. mined experimentally. The value of $f_{h}$ was used to calculate the horizontal stiffness $\left(k_{h}\right)$ of the loaded rubber using the equation

$$
k_{h}=m\left(2 \pi f_{h}\right)^{2} .
$$

Since the model used here was a thin slice through the real system, a fictitious density had to be assigned to the modeled aluminum plate to give the correct loading conditions per unit area of rubber support. The shear modulus $(G)$ and the Young's modulus $(E)$ of the aluminum were also scaled up by the same factor as the density in order to maintain the stiffness properties of the plate. The damping of the internal modes of the plate was modeled using a "structural element damping coefficient" facility in the NASTRAN package. ${ }^{4}$ For initial models the damping was chosen so that the $Q$ 's of the internal modes of the aluminum plate were $\sim 20$, while for later ones the modes were critically damped $(Q \sim 0.5)$.

Further, since one slice-like rubber support in the model represented two block-like supports in the real system, the moduli of the rubber were scaled to give the stack realistic stiffness properties. The shear modulus of the rubber was determined using the equation

$$
G=k_{h} h / A
$$

where $h$ is the height of the rubber supports, $A$ is the total top surface area of the rubber, and then scaled to compensate for the reduction in area $A$.

There is approximately no volume change when rubber undergoes tension or compression, ${ }^{7}$ hence Poisson's ratio $v \sim 0.5$. The value of Young's modulus for the rubber was calculated by the finite element program according to the relationship:

$$
E=2 G(1+v)
$$

giving

$$
E \sim 3 G .
$$

A structural element damping coefficient was assigned to the rubber so that the internal modes of the rubber supports were damped to give an internal $Q$ of $\sim 5$, this being a typical observed value. ${ }^{8}$ Viscous damping of the fundamental stack resonances was incorporated by connecting the nodes at the bases and tops of the rubber supports with orthogonally connected damping elements in 


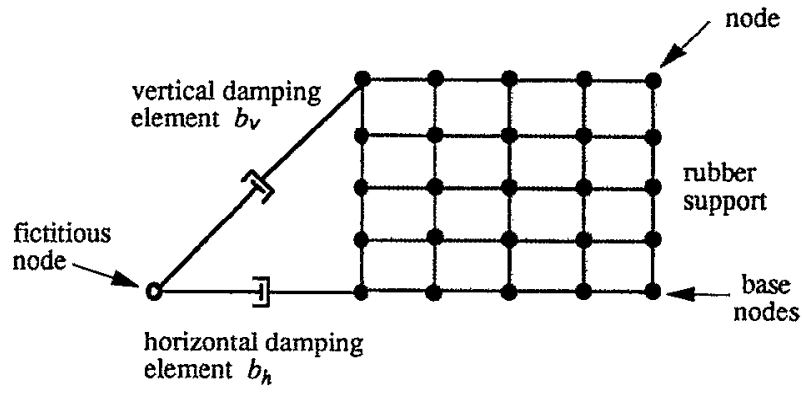

FIG. 3. Damping elements are connected across each rubber support to provide viscous damping of the supported plate to the ground in both the horizontal and vertical directions. To show the damping elements clearly, they are not drawn at right angles to each other. Each pair of horizontal and vertical elements are connected orthogonally using a massless "fictitious node." Using "multipoint constraints" (Ref. 3) this node can be instructed to follow the vertical motion of the horizontal element and the horizontal motion of the vertical element. In this way the levels of damping in the two directions can be independently defined. Note that each pair of nodes along the base and top of a given support are connected in this way.

the horizontal and vertical directions (see Fig. 3). The size of the horizontal damping factor $b_{h}$ was established using the equation

$$
b_{h}=2 \pi m f_{h} / Q_{h} \text {, }
$$

where $Q_{h}$, the corresponding quality factor, was chosen to be $\sim 5$ as suggested by some elementary experimental tests. These experiments suggested that the $Q$ of the vertical mode of each stage would be somewhat higher than of the horizontal mode. Thus the size of the damping factor for vertical motion, $b_{v}$, was calculated to give $Q_{v} \sim 15$. Note that the vertical stiffness and viscous damping of the rubber supports also determines the tilting resonant frequency and corresponding quality factor. Further details of this finite element model are given in Ref. 6.

Despite the approximate nature of this model it incorporates the essential features of each stage of the stack and should lead to a reasonable understanding of the performance and an indication of the cross-coupling mechanisms which take place in multistage vibration isolation stacks.

\section{METHODS OF ANALYSIS}

Eigenvalue analyses were carried out on the single and two-stage models illustrated in Fig. 4. The horizontal to horizontal and vertical to vertical frequency response analyses for each model were carried out by driving the base nodes of the rubber supports (see Fig. 3) sinusoidally in the relevant direction with unit amplitude displacement at all frequencies in the range considered. In each case the resulting amplitudes of motion of the centers of mass of the supported plates in the appropriate directions were observed to give a value for the transmissibility at each frequency. To investigate the isolation for tilting motion the base nodes were driven with a unit vertical input at the end nodes of the base of the rubber supports and with progressively decreasing displacement towards the center of the plate as illustrated in Fig. 5. The amplitudes of tilting mo-
ONE - STAGE STACK MODEL

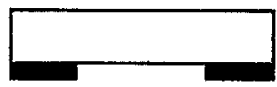

balanced rubber

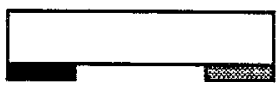

imbalanced rubber
TWO - STAGE STACK MODEL

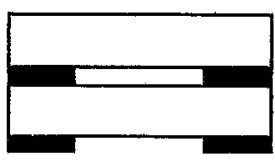

balanced rubber

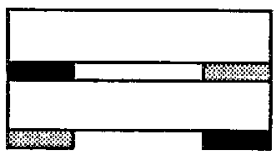

asymmetrically imbalanced rubber

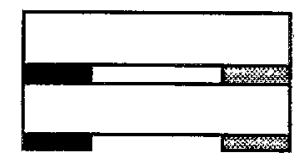

symmetrically imbalanced rubber
FIG. 4. The various stack systems investigated.

tion of the plates about their centers of mass were evaluated in each case (at frequencies below the internal resonances of the plates) by noting the relative vertical displacements of the end and center nodes of the plates.

Investigations into the cross coupling of tilting ground motion to horizontal and vertical motion of the centers of mass of the plates were carried out using the same progressive driving mechanism described above. The amplitudes of the horizontal or vertical motion of the centers of mass of the plates were compared to the angle of tilt at the base of the stack to give a value for the transmissibility in units of $\mathrm{m} / \mathrm{rad}$.

In the analysis of the remaining cross-coupling effects, unit vertical or horizontal input at the driven base nodes was used and the orthogonal directional components at the center of mass nodes of the plates were noted. The amplitude of tilting motion of the plates about their centers of mass wcrc also evaluated in each case to give the transmissibility in units of $\mathrm{rad} / \mathrm{m}$.

\section{EIGENVALUE ANALYSES}

The one-stage stack model was constrained to move in the $x-z$ plane. Therefore only two of the three possible translational degrees of freedom remained. Similarly only

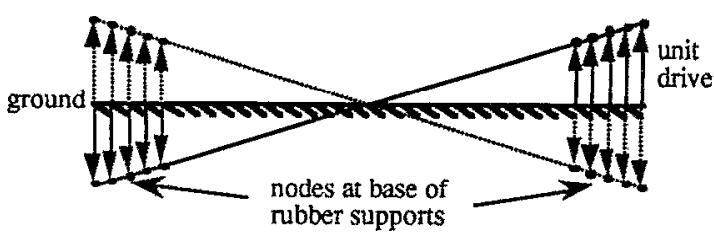

FIG. 5. Progressive driving mechanism for investigations of couplings of tilting motions. 


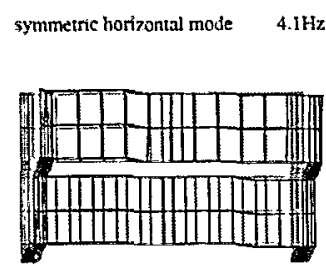

antisymmetric horizontal mode $10.8 \mathrm{~Hz}$

symmetric vertical mode $\quad 8.3 \mathrm{~Hz}$

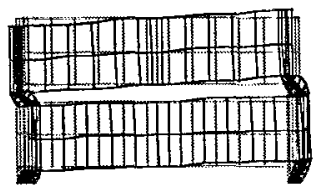

antisymmetric vertical mode $\quad 21.7 \mathrm{~Hz}$
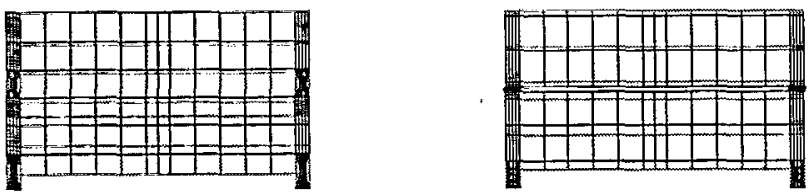

symmetric tilting mode

$13.9 \mathrm{~Hz}$

antisymmetric tilting mode $\quad 35.6 \mathrm{~Hz}$
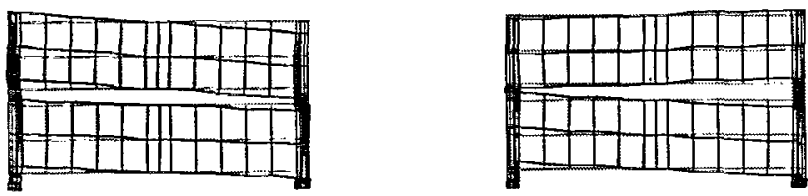

FIG. 6. Modes present in the two-stage stack with balanced rubber properties. The modes occur in pairs corresponding to the oscillations in the two stages being in or out of phase with each other. Note that the horizontal and tilting modes are not pure but are in fact weakly coupled together, as can be seen in the figure. The step-like nature of the displacements is an artifact of the plotting method used.

one rotational degree of freedom remained unconstrained, this being tilting motion about the $y$ axis (refer to Fig. 1). As a consequence of these constraints the modeled stack had only three fundamental modes of oscillation, namely, the horizontal, vertical, and tilting modes. With rubber of identical stiffness properties on each side of the stack the fundamental frequencies were at $f_{h} \sim 7 \mathrm{~Hz}, f_{v} \sim 13 \mathrm{~Hz}$, and $f_{t} \sim 22 \mathrm{~Hz}$. (These values agreed reasonably well with those predicted using simple theory.) The lowest internal mode of the metal plate was at $\sim 2 \mathrm{kHz}$.

The first six normal modes associated with the twostage stack having balanced rubber properties are shown in Fig. 6. The dotted lines represent the undisturbed position of the mesh for reference. These diagrams were generated using the graphics package FEMVIEW. ${ }^{9}$

With a $10 \%$ reduction in the stiffness of the rubber on one side of the stack, the computed fundamental stack frequencies were slightly lower in value, as one would expect, and the mode shapes were asymmetrical about the $y-z$ plane.

A complete summary of the fundamental frequencies obtained for the various stack models analyzed is given in Fig. 7.

\section{TRANSMISSIBILITY OF ONE- AND TWO-STAGE STACKS}

The transmissibility of the one-stage stack was analyzed for a number of possible couplings.

(a) Vertical drive to vertical response; Horizontal drive to horizontal response; Tilt drive to tilt response. These are

\begin{tabular}{|c|c|c|c|c|c|c|}
\hline \multicolumn{7}{|c|}{ one-stage stack } \\
\hline MODEL & \multicolumn{2}{|c|}{$f_{k}$} & \multicolumn{2}{|c|}{$f_{v}$} & \multicolumn{2}{|c|}{$f_{t}$} \\
\hline balanced & \multicolumn{2}{|c|}{6.7} & \multicolumn{2}{|c|}{13.4} & \multicolumn{2}{|c|}{22.1} \\
\hline imbalanced & \multicolumn{2}{|c|}{6.6} & \multicolumn{2}{|c|}{13.1} & \multicolumn{2}{|c|}{21.5} \\
\hline \multicolumn{7}{|c|}{ two-stage stack } \\
\hline MODEL & $f_{h}^{s}$ & $f_{h}^{a}$ & $f_{v}^{s}$ & $f_{v}{ }^{a}$ & $f_{t}^{s}$ & $f_{t}^{a}$ \\
\hline balanced & 4.1 & 10.8 & 8.3 & 21.7 & 13.9 & 35.6 \\
\hline $\begin{array}{l}\text { symmetrically } \\
\text { imbal:anced }\end{array}$ & 4.0 & 10.5 & 8.1 & 21.1 & 13.6 & 34.7 \\
\hline $\begin{array}{l}\text { asymmetrically } \\
\text { imbalanced }\end{array}$ & 4.0 & 10.5 & 8.1 & 21.2 & 13.5 & 34.7 \\
\hline
\end{tabular}

FIG. 7. Summary of the fundamental resonant frequencies for the various stack systems investigated. All frequencies are given in hertz. Superscripts denote symmetric $(s)$ or antisymmetric $(a)$ mode. Subscripts denote horizontal $(h)$, vertical $(v)$, or tilt $(t)$. Imbalanced figures are for a $10 \%$ reduction in the rubber stiffness on one side of each stage.

due to direct coupling of the displacements through the mass/spring (plate/rubber) arrangement.

(b) Horizontal drive to tilt response; Tilt drive to horizontal response. The first type of coupling is due to the effective torques on the supported plate (Fig. 8). One of the torques results from the line of action of the horizontal force exerted by the top of the rubber on the base of the metal plate being offset vertically from the center of mass of the plate. The other torque, acting across the top of each rubber support results from the distortion of the rubber.

The second type of coupling is due to the geometrical effect of the vertical offset of the center of mass of the plate from the axis of tilt (Fig. 9).

(c) Tilt drive to vertical response; Vertical drive to tilt response; Vertical drive to horizontal response; Horizontal drive to vertical response. For the linear analysis used in this work these occur only when the rubber stiffness is imbalanced and effectively arise due to torques exerted on the supported plates by the rubber pads. There are of course tilt to vertical and horizontal to vertical cross couplings when the rubber stiffness is balanced; these are secondorder effects producing responses at frequencics other than the driving frequency, and since seismic noise has a relatively small amplitude these couplings are not generally

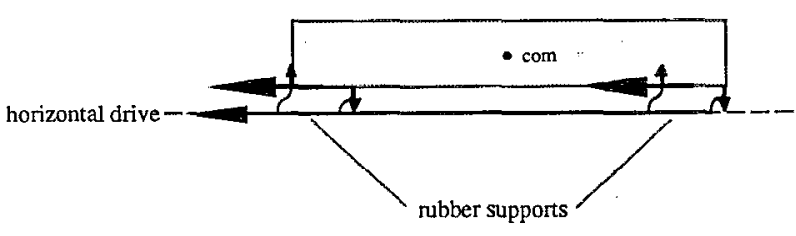

FIG. 8. Mechanism for the cross coupling of horizontal to tilting motion in a one-stage stack. The plate is subject to torques which force it to rotate about its center of mass (com). One of the torques results from the shear force of the rubber acting on the metal plate being vertically offset from the center of mass of the plate. The other torque, acting across the top of each rubber support, results from the distortion of the rubber. 


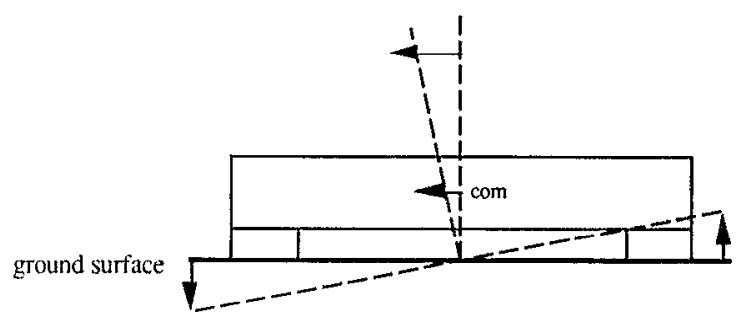

FIG. 9. Mechanism for the cross coupling of tilt to horizontal motion in a one-stage stack. At low frequencies the system is essentially rigid and the center of mass (com) of the plate is driven linearly in the horizontal direction. The larger the vertical offset the larger this effect.

significant. The results of these various analyses are shown in Figs. 10-17. In Fig, 10, the transmissibility curve for vertical drive to vertical response, the aluminum plate was modeled to have a $Q$ of 20 and the effect of the lowest plate resonance can easily be seen. It should be notcd that for the three-dimensional stack shown in Fig. 1 the first plate resonance will be at a lower frequency than that of the twodimensional model presented here. As such plate resonances compromise the isolation significantly and are liable to appear at frequencies of interest for gravitational wave detection it is important that they are well damped. In the remaining two-dimensional analyses, in order to simplify the situation it was assumed that the plates were critically damped $(Q=0.5)$. The effect of this damping is also shown in Fig. 10.

The form of each transmissibility curve in Figs. 10-17 can be understood relatively easily from consideration of the dynamics of the system. In situations where a single resonance of the mass/rubber stage is dominant the transmissibility falls at approximately $12 \mathrm{~dB}$ per octave above the resonance at $f_{0}$ up to a frequency $Q f_{0}$, where $Q$ is the quality factor of the resonance, and at $6 \mathrm{~dB}$ per octave above this frequency. Where there are two resonances in-

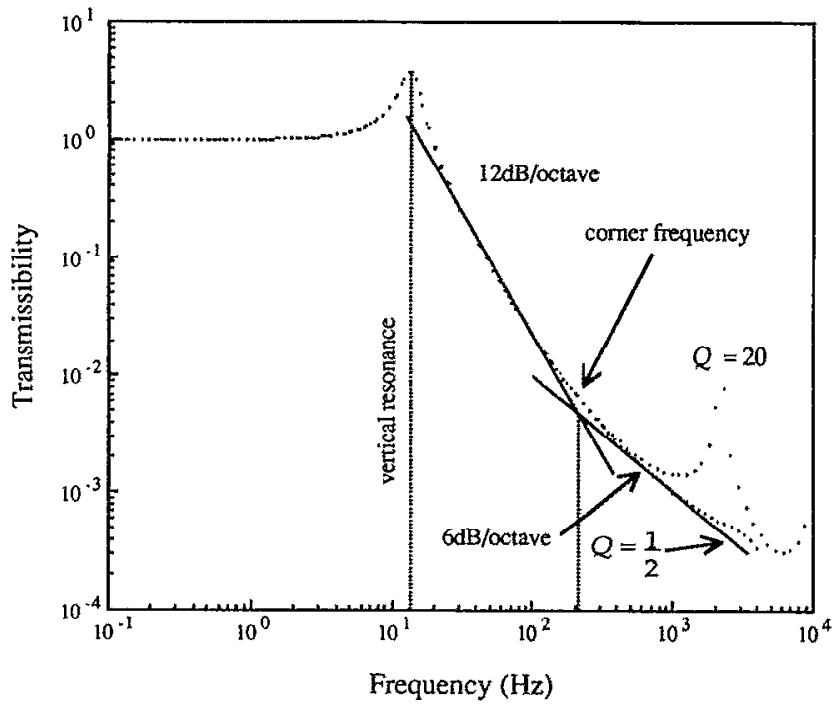

FIG. 10. Vertical drive to vertical response in the one-stage stack.

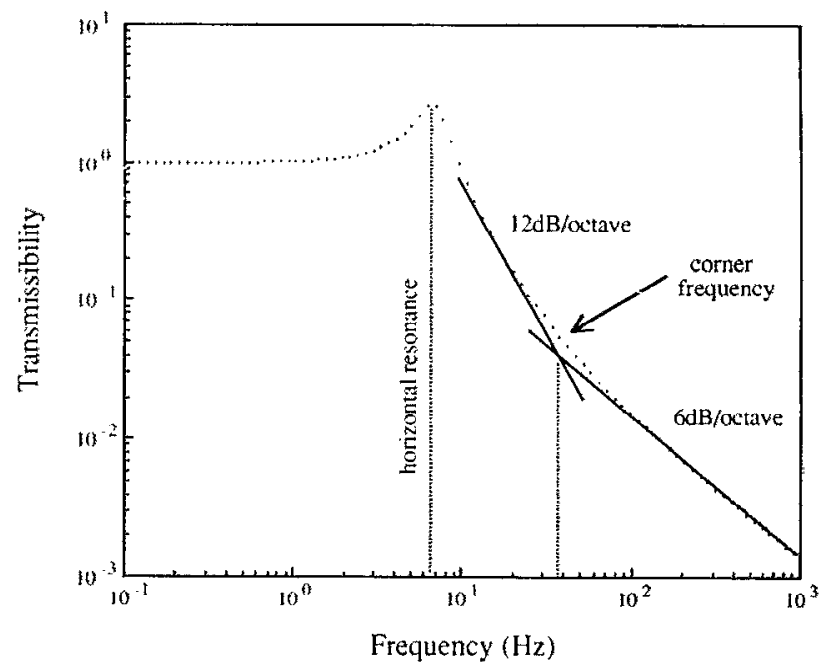

FIG. 11. Horizontal drive to horizontal response in the one-stage stack.

volved and where these resonances are effectively in series, e.g., in going from tilt drive to horizontal response (Fig. 13), the transmissibility falls at twice this rate. In some cases, at high frequency, the transmissibility curve flattens out as a result of computational rounding effects discussed more fully below.

It should be noted that certain of the couplings between directions disappear if the geometry can be arranged to remove the vertical offsets mentioned above. However such arrangements are potentially difficult to realize. The magnitude of coupling in set (c) described above depends on the magnitude of the imbalance of the stiffness of the rubber pads. For convenience a summary table of the transmissibility values obtained for the one-stage stack at a frequency of $100 \mathrm{~Hz}$ is given in Fig. 18 .

The situation with a two-stage stack is somewhat more complicated since there are a larger number of degrees of

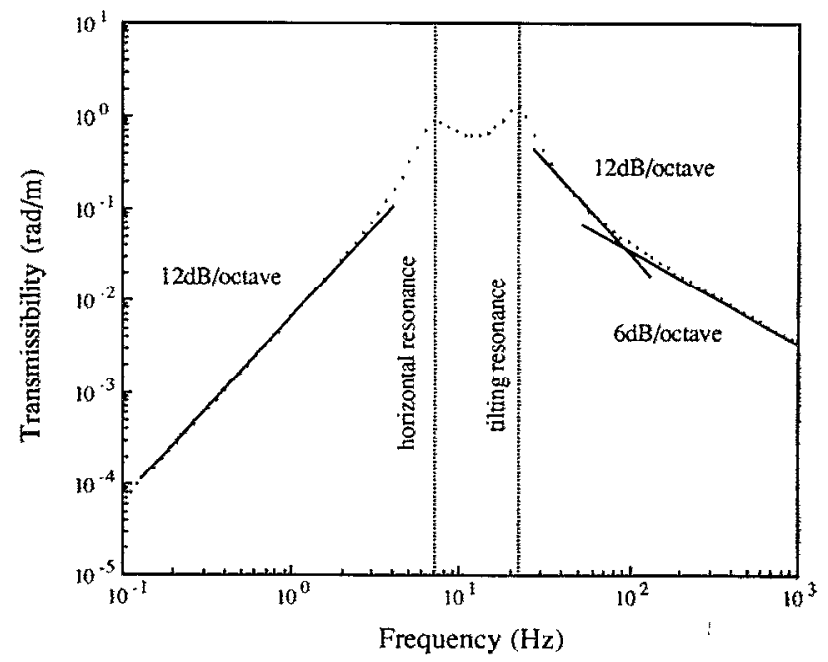

FIG. 12. Horizontal drive to tilt response in the one-stage stack. 


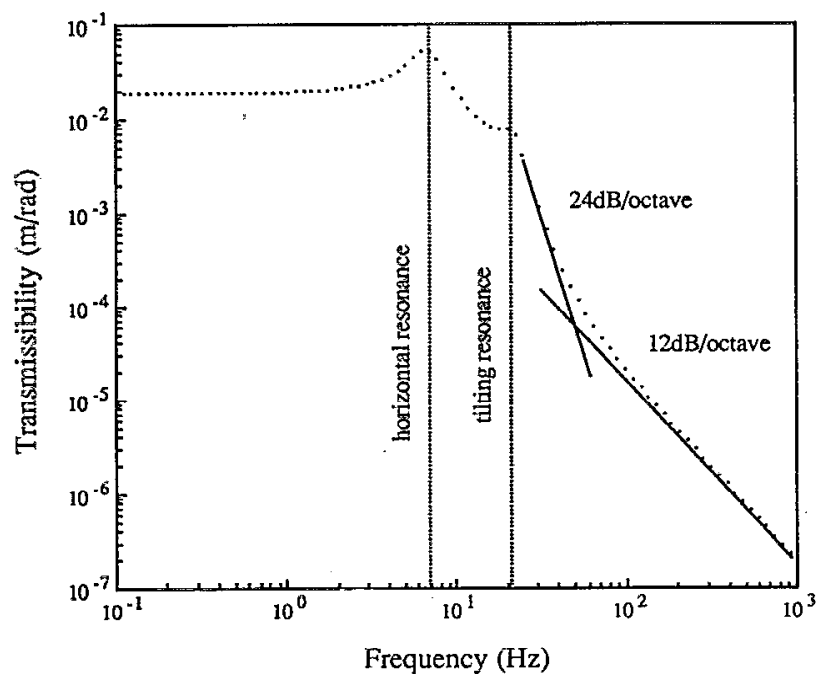

FIG. 13. Tilt drive to horizontal response in the one-stage stack.

freedom and various competing routes for the transmission of motions exist. Consider, for example, the case of horizontal response of the second plate driven by horizontal motion of the base. The largest effect, as one would expect, is due to the horizontal motion of the base exciting horizontal motion in the first plate, which in turn excites horizontal motion of the second plate. There are other effects, however, such as horizontal base motion exciting tilt of the first plate which leads to horizontal motion of the second plate.

Some of the results for the arrangements of two-stage stacks given in Fig. 4 are shown in Figs. 19-25, and again these can be understood from the dynamics of the system. In most cases the response at the first plate is similar to that for the single stage stack and the second stage provides extra isolation as would be intuitively expected. However

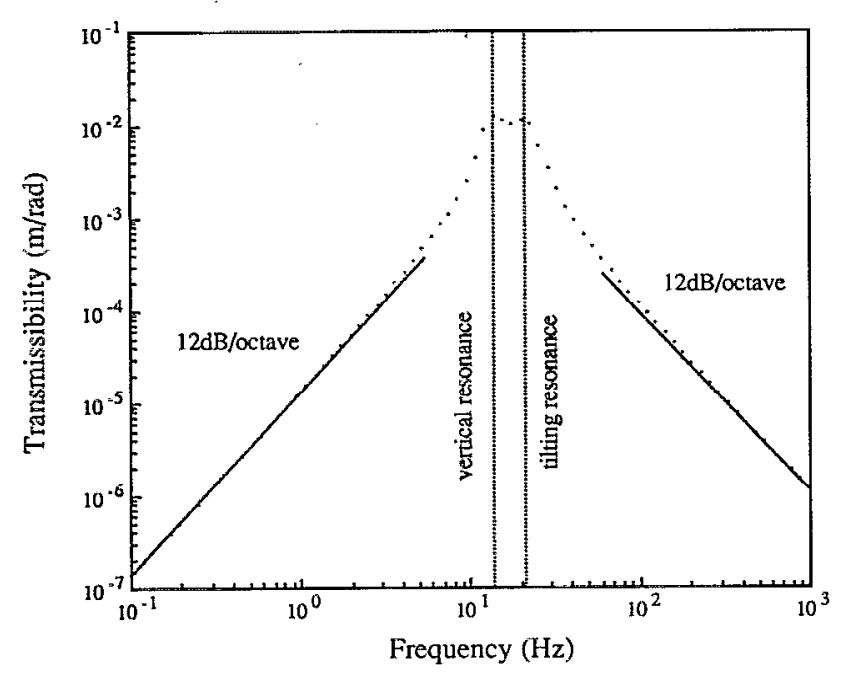

FIG. 14. Tilt drive to vertical response in the one-stage stack with a $10 \%$ stiffness imbalance in the rubber springs.

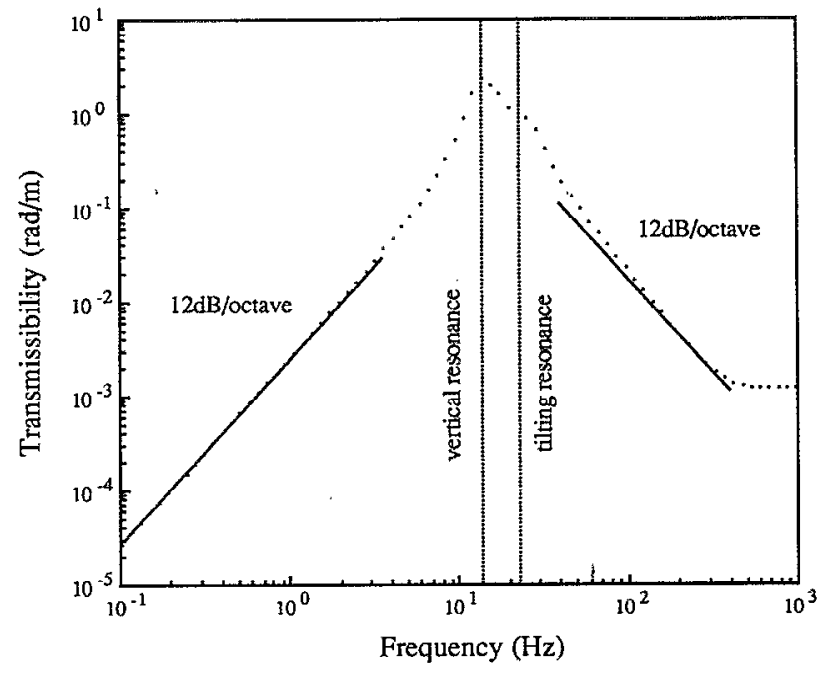

FIG. 15. Vertical drive to tilt response in the one-stage stack with a $10 \%$ stiffness imbalance in the rubber springs.

there are three exceptions to this, and these result from the dynamics involved in the transmission route which dominates:

(1) Tilt to horizontal transmissibility (Fig. 21): Tilt drive (base) to horizontal response (center of mass 2) comes about from tilting motion (base) to tilting motion (center of mass 1) followed by conversion to horizontal motion at the top of mass 1 (intraplate conversion) and then to horizontal motion (center of mass 2 ).

(2) Vertical to horizontal transmissibility (Fig. 24): Vertical drive (base) to horizontal response (center of mass 2) comes about from vertical motion (base) to tilting motion (center of mass 1) to horizontal motion (top of mass 1) to horizontal motion (center of mass 2).

(3) Horizontal to vertical transmissibility (Fig. 25): Horizontal drive (base) to vertical response (center of

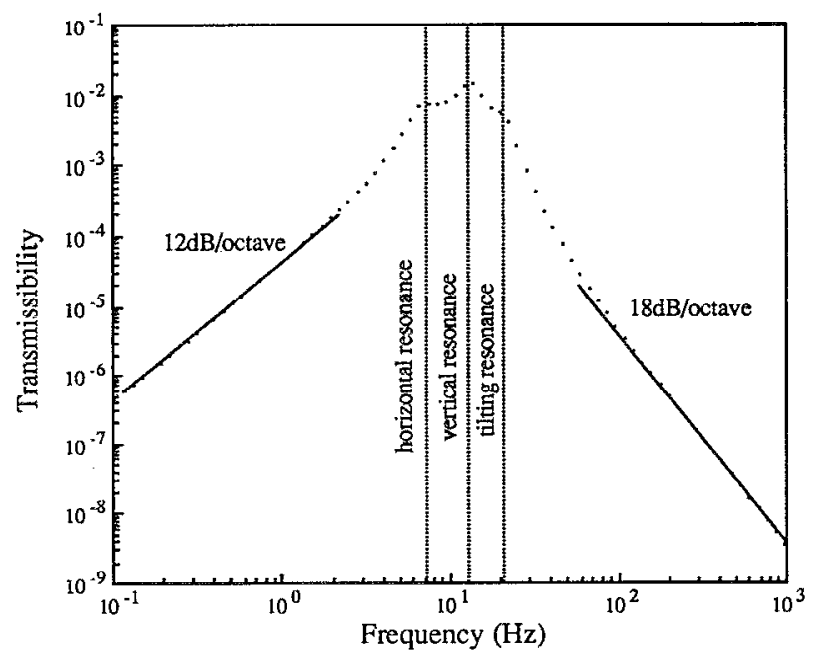

FIG. 16. Vertical drive to horizontal response in the one-stage stack with a $10 \%$ stiffness imbalance in the rubber springs. 


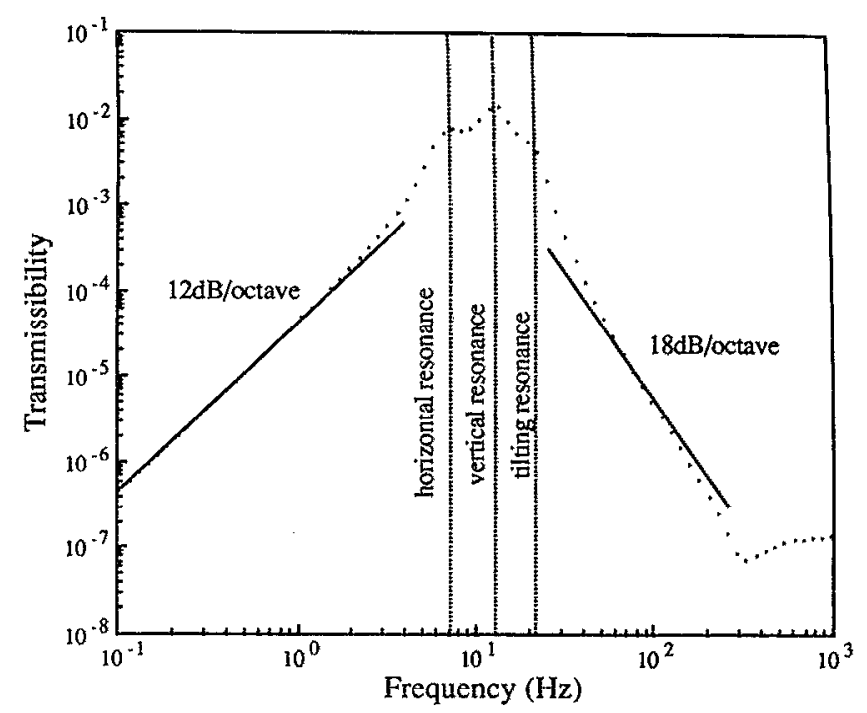

FIG. 17. Horizontal drive to vertical response in the one-stage stack with a $10 \%$ stiffness imbalance in the rubber springs.

mass 2) arises from horizontal motion (base) to tilting motion (center of mass 1) to vertical motion (center of mass 2).

Other interesting factors emerge from these analyses. For example in the case of vertical drive (base) to horizontal response (center of mass 2 ) the horizontal motion at stage 1 is smaller than that at stage 2 (Fig. 24). This arises from a balancing of the restoring forces acting at plate 1 due to the presence of stage 2 above it. In most cases for the two-stage system it does not matter whether the $10 \%$ imbalance in rubber properties is symmetrical or asymmetrical for the two stages. An exception to this is the response of stage 1 in the case of horizontal drive (base) to vertical response (center of mass 2 ). Figure 25 shows the situation for symmetrically imbalanced rubber where there is little dynamical balancing for stage 1 . Even with the presence of the overlying stage there remains a difference in

\begin{tabular}{|c|c|c|c|}
\hline IN & HORIZONTAL & VERTICAL & TILT \\
\hline HORIZONTAL & $\sim 1.6 \times 10^{-2}$ & $-5.0 \times 10^{-6}$ & $\sim 4.4 \times 10^{-2} \mathrm{rad} / \mathrm{m}$ \\
\hline VERTICAL & $-5.1 \times 10^{-6}$ & $-2.3 \times 10^{-2}$ & $-2.3 \times 10^{-2} \mathrm{rad} / \mathrm{m}$ \\
\hline TILT & $-2.1 \times 10^{-5} \mathrm{~m} / \mathrm{rad}$ & $-1.2 \times 10^{-4} \mathrm{~m} / \mathrm{rad}$ & $\sim 6.4 \times 10^{-2}$ \\
\hline
\end{tabular}

FIG. 18. Summary of the various transmissibility values at $100 \mathrm{~Hz}$ for the one-stage stack. Figures in bold are given for a $10 \%$ stiffness imbalance in the rubber springs.

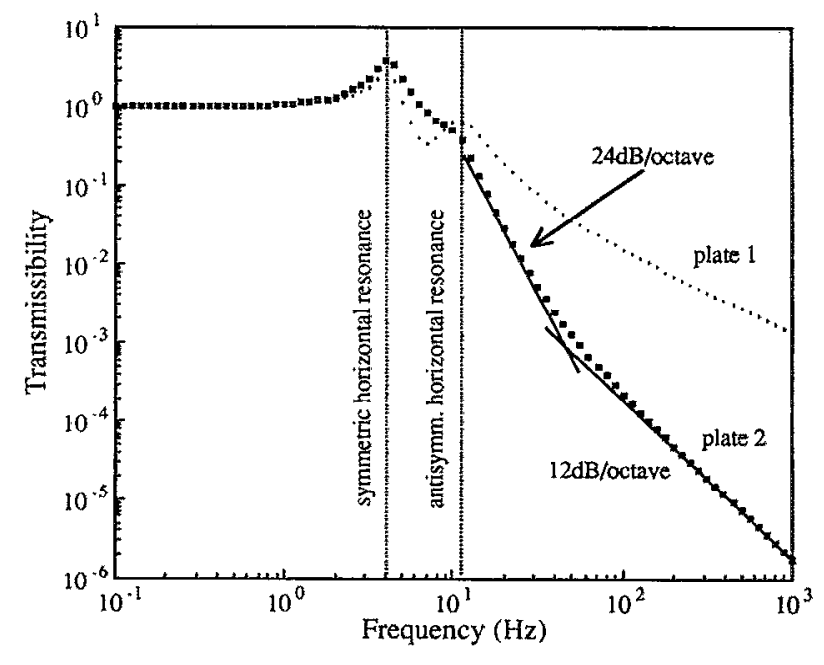

FIG. 19. Horizontal drive to horizontal response in the two-stage stack.

the vertical stiffness at each end of plate 1. Its induced tilting motion will therefore not be about its center of mass and will lead to vertical movement of the center of mass. For asymmetrically imbalanced rubber there is a dynamical balancing effect on stage 1 which reduces this vertical movement by a large factor.

\section{EXTENSION TO THE UNDERSTANDING OF A FOUR-STAGE STACK}

It is clearly possible to carry out the same types of analyses for multiple stage stacks. However, in the work described above we encountered two numerical problems. One was due to the fact that the stiffnesses of the aluminum and rubber are very disparate. The other problem was seen at high frequencies where the degree of isolation is such that the output is very small compared to the input. The first problem manifests itself in error messages from NASTRAN and leads to potential inaccuracies in the results. At the suggestion of the NASTRAN vendors we did

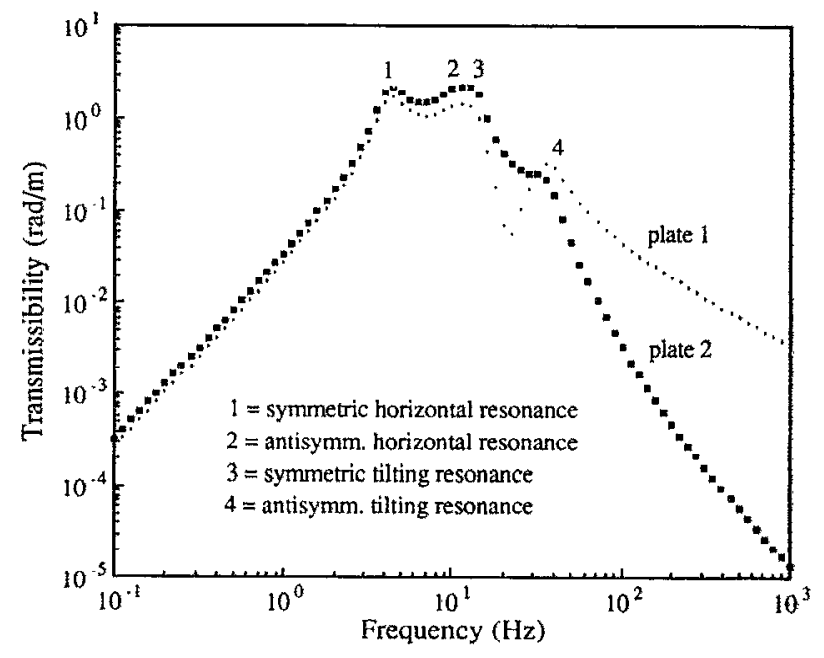

FIG. 20. Horizontal drive to tilt response in the two-stage stack. 


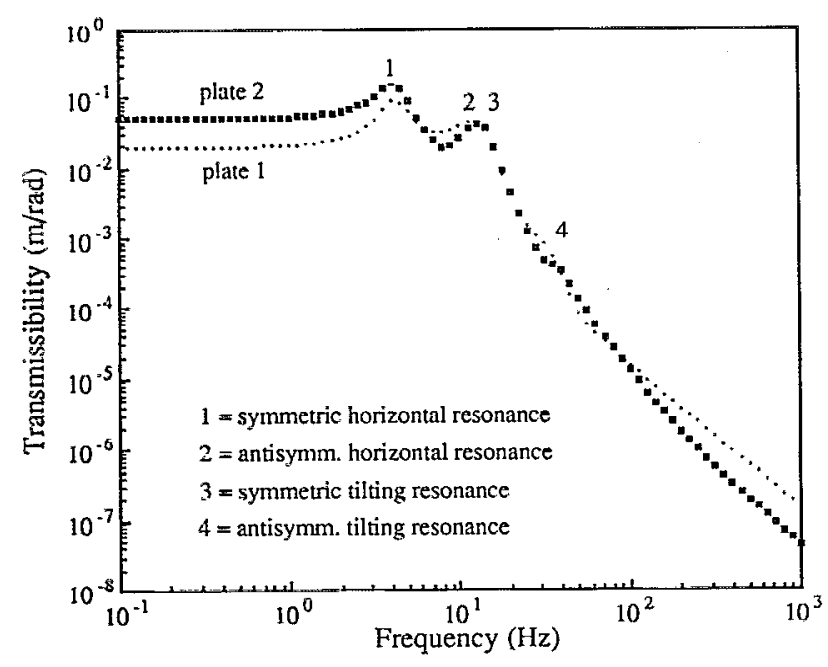

FIG. 21. Tilt drive to horizontal response in the two-stage stack.

not discard results when error messages occurred, but took care to examine the results carefully for the kind of inaccuracies characteristic of such problems. The second problem manifested itself as a flattening out of transmissibility at a value of around $10^{-7}$ or lower. This effect was reduced to this acceptable level by using the "direct" rather than "modal" method of calculating transmissibilities. ${ }^{4}$ However the direct method becomes very demanding in computing time for models of more than two stages.

Fortunately, from considerations of the mechanisms involved in the transmissibility of one- and two-stage stacks, it is possible to predict relatively easily the main features of the isolation performance of a stack with more stages. For example, the principal transmission routes for both the vertical and horizontal responses of a four-stage stack are shown in Figs. 26 and 27, and the performance at $100 \mathrm{~Hz}$ in each case has been calculated. It is clear from these results that by using four stages of the particular

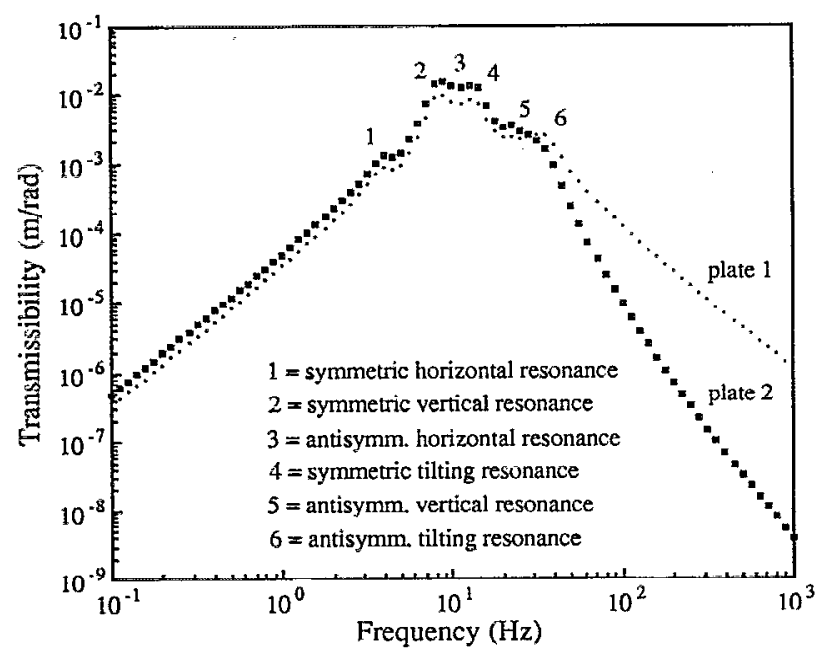

FIG. 22. Tilt drive to vertical response in the two-stage stack with a $10 \%$ stiffness imbalance in the rubber springs.

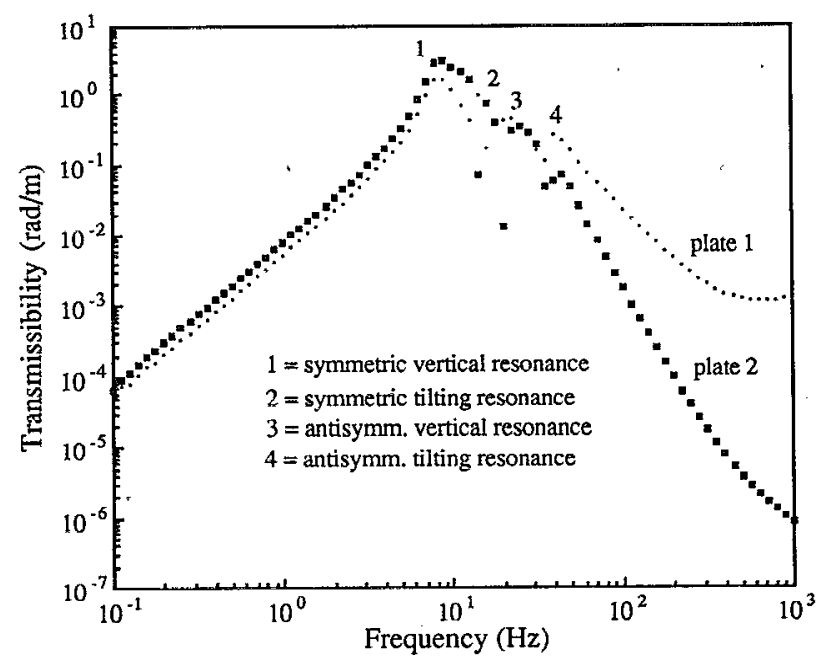

FIG. 23. Vertical drive to tilt response in the two-stage stack with a $10 \%$ stiffness imbalance in the rubber springs.

design considered here, sufficient vertical isolation can be achieved and that the horizontal isolation is much better than required for our particular purpose. (If there were much less cross coupling of vertical to horizontal motion occurring in the subsequent pendulum suspension, less vertical isolation would be required and the number of stages required in the stack could be reduced.)

We assume that the stages have the dimensions used earlier but in order to generalize the analyses we allow the possibility of embedding the bases of the rubber supports into the underlying aluminum plates to control the degree of conversion of tilting motion of the center of mass of a plate to horizontal motion at the base of the rubber (intraplate conversion). The relevant variable here is $a$, the vertical distance between the center of mass of a metal plate and the level above it at which the rubber contacts the plate. We also allow for the fact that the suspension point of the pendulum system mounted from the stack may

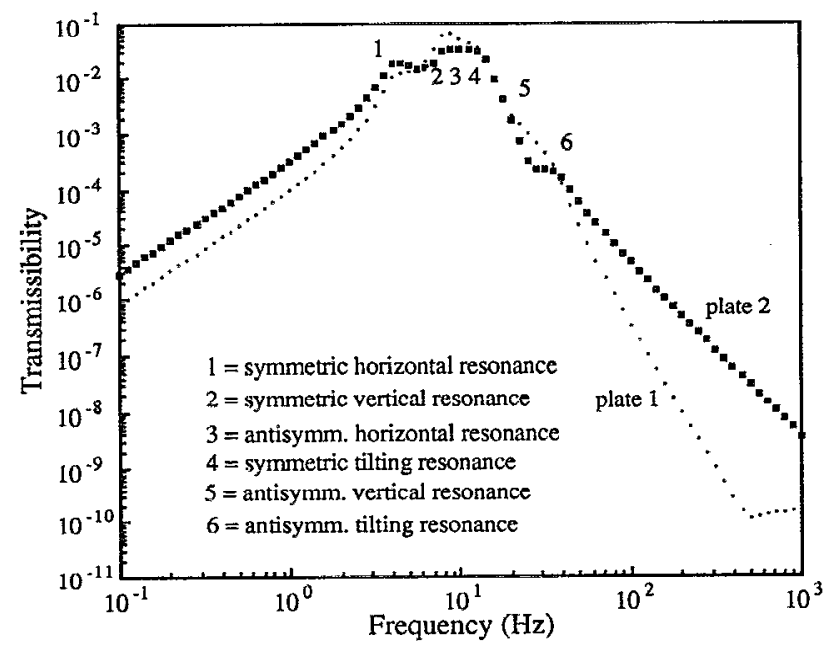

FIG. 24. Vertical drive to horizontal response in the two-stage stack with a $10 \%$ stiffness imbalance in the rubber springs. 


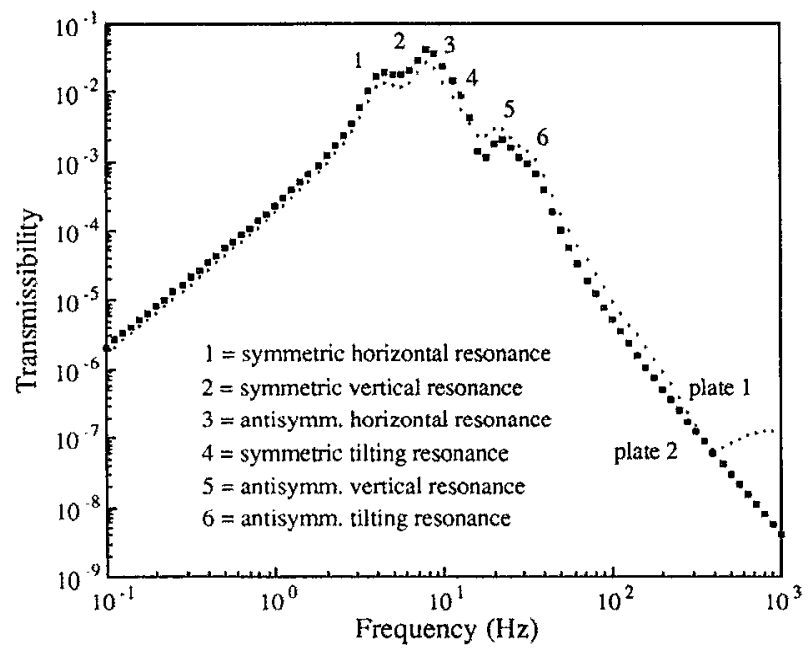

FIG. 25. Horizontal drive to vertical response in the two-stage stack with a $10 \%$ stiffness imbalance in the rubber springs.

be displaced a vertical distance $z$ and a horizontal distance $x$ from the center of mass of the top plate. It is interesting to see that for the dimensions chosen the most important route for the vertical motion is the simplest route, i.e., by direct vertical coupling from stage to stage. However in the horizontal case the simplest type of coupling up the stack is not the most important mechanism. For the four-stage stack studied the most important mechanism (route 2) involves intraplate conversion, but the effect of this may be greatly reduced by allowing the rubber springs to be embedded into the underlying plates, especially near the top of the stacks. Assuming this is done, there remains another important transmission route for introducing horizontal motion to the pendulum suspension point. This is direct transmission of tilts up the stack and linear conversion to horizontal movement by the vertical offset of the suspension point. Reduction of this coupling to a level such that the noise introduced will be less than that introduced by the simplest horizontal transmission route will require very careful adjustment of the suspension point.

It is worth recalling from Fig. 8 that in a single stage the conversion of horizontal movement into tilt comes about from two apparently separate torques exerted on the plate. Simple dynamical analysis shows that these are related to each other and cancel each other out to give no tilt if the center of mass of the plate is arranged to lie on a line passing through the midpoints of the rubber supports. ${ }^{10}$ Unfortunately simplicity of design is lost if we wish to extend this to a multiple stage stack arrangement and cut out horizontally driven tilts, as the center lines of the rubber supports for each stage would have to be coincident. Fortunately it would seem that it is not very important to do this for our particular application.

\section{DISCUSSION}

From the finite element analysis of one- and two-stage stack systems and the extension of these findings to a fourstage stack it is clear that the simple dynamical models often may not be adequate as they do not account for some potentially important routes for noise transmission. However the analyses do suggest that performance of the order required for gravitational wave detectors should be attainable. Particularly high performance is achievable if care is taken in the mounting of the rubber at stages close to the top of the stack to reduce intraplate conversion, and if care is taken in reducing the separation in the vertical and horizontal directions of the suspension point of the pendulum from the center of mass of the top plate.

It should be pointed out that gravity was not included in this work. Its inclusion may affect overall static stability and would probably have some dynamic effects, such as the increased loading on the lower stages of the stack causing changes of stiffness of the rubber and hence changes in the resonant frequencies of the lower stages. However provided attention is paid to maintaining static stability, and provided any changes in rubber stiffness are allowed for at

\begin{tabular}{|c|c|c|}
\hline transmission route & transmissibllity $(T)$ at $100 \mathrm{~Hz}$ & $\begin{array}{l}\text { vertical motion }(d z) \text { of } \\
\text { suspension point at } 100 \mathrm{~Hz}\end{array}$ \\
\hline $1 \quad v_{\text {buse }}-v_{\text {com } 1}-v_{c o m 2}-v_{c o m 3}-v_{c o m}\left(v_{s p^{\prime}}\right)$ & $\sim 2.8 \times 10^{-7}$ & $\sim 2.8 \times 10^{-18} \mathrm{~m} / \sqrt{\mathrm{Hz}}$ \\
\hline $2 \quad t_{\text {buse }}-t_{\operatorname{com} 1}-t_{\mathrm{com} 2}-t_{\mathrm{com} 3}-\left[\mathrm{tcom}_{\mathrm{com}}-\mathrm{v}_{\mathrm{sp}}\right]$ & $\sim 3.4 \times 10^{-8}\left[\frac{x}{2.0 \times 10^{-3}}\right] \mathrm{m} / \mathrm{rad}$ & $\sim 6.8 \times 10^{-19}\left[\frac{x}{2.0 \times 10^{-3}}\right] \mathrm{m} / \sqrt{\mathrm{Ez}}$ \\
\hline $3 t_{\text {tbese }}-t_{c o m 1}-t_{c o m} 2-t_{c o m} 3-v_{c o m} 4\left(v_{s p}\right)$ & $\sim 3.1 \times 10^{-8} \mathrm{~m} / \mathrm{rad}$ & $\sim 6.2 \times 10^{-19} \mathrm{~m} / \sqrt{\mathrm{Hz}}$ \\
\hline $4 \quad h_{\text {base }}-t_{\operatorname{con} 1}-t_{\operatorname{com} 2}-t_{\operatorname{com} 3}-\left[t_{\operatorname{com} 4}-v_{s p}\right]$ & $\sim 2.3 \times 10^{-8}\left[\frac{x}{2.0 \times 10^{-3}}\right]$ & $\sim 2.3 \times 10^{-19}\left[\frac{x}{2.0 \times 10^{-3}}\right] \mathrm{m} / \sqrt{\mathrm{Hz}}$ \\
\hline
\end{tabular}

FIG. 26. The main transmission routes predicted to give rise to vertical motion at the top of the four-stage stack. Horizontal, vertical, and tilting motions are denoted by $h, v$, and $t$, respectively. Round brackets indicate that the motion of the pendulum suspension point (sp) is equivalent to that of the center of mass (com) of the top plate of the stack. Square brackets are used where cross coupling of motions occurs within a metal plate. Here $x$ is the horizontal offset between the pendulum suspension point and the center of mass of the top plate of the stack. We have used an offset $x$ of $2 \mathrm{~mm}$ as the reference offset since this would be a reasonable estimate of the accuracy to which the top plate center of mass and suspension point might be made coincident. The + symbol in route 3 indicates that a $10 \%$ stiffness imbalance exists in the rubber springs of stage 4 . 


\begin{tabular}{|c|c|c|}
\hline transmission route & transmissibility $(T)$ at $100 \mathrm{~Hz}$ & $\begin{array}{l}\text { horizontal motion }(d x) \text { of } \\
\text { suspension point at } 100 \mathrm{~Hz}\end{array}$ \\
\hline $1 h_{\text {base }}-h_{\text {com } 1}-h_{\text {com } 2}-h_{\text {com } 3}-h_{\text {com } 4}\left(h_{\text {sp }}\right)$ & $\sim 6.6 \times 10^{-8}$ & $\sim 6.6 \times 10^{-19} \mathrm{~m} / \sqrt{\mathrm{Hz}}$ \\
\hline $\left.2 t_{b a s e}-t_{c o m 1}-t_{c o m} 2-\left[t_{c o m} 3-h_{\text {top } 3}\right]-h_{c o m}\left(h_{s p}\right)\right]$ & $\sim 5.5 \times 10^{-8}\left[\frac{a}{1.3 \times 10^{-2}}\right]^{\mathrm{m} / \mathrm{rad}}$ & $\sim 1.1 \times 10^{-18}\left[\frac{a}{1.3 \times 10^{-2}}\right] \mathrm{m} / \sqrt{\mathrm{Hz}}$ \\
\hline $3 t_{\text {base }}-t_{\text {com1 } 1}-\left[t_{\text {com2 }}-h_{\text {top } 2}\right]-h_{\text {com } 3}-h_{\text {com } 4}\left(h_{s p}\right)$ & $\sim 1.4 \times 10^{-8}\left[\frac{a}{1.3 \times 10^{-2}}\right]^{\mathrm{m} / \mathrm{rad}}$ & $\sim 2.8 \times 10^{-19}\left[\frac{a}{1.3 \times 10^{-2}}\right]^{\mathrm{m} / \sqrt{\mathrm{Hz}}}$ \\
\hline $4 h_{b a s e}-t_{c o m 1}-t_{c o m} 2-\left[t_{c o m} 3-h_{\text {top } 3}\right]-h_{c o m}\left(h_{s p}\right)$ & $\sim 3.7 \times 10^{-8}\left[\frac{a}{1.3 \times 10^{-2}}\right]$ & $\sim 3.7 \times 10^{-19}\left[\frac{a}{1.3 \times 10^{-2}}\right]^{m / \sqrt{\mathrm{Hz}}}$ \\
\hline${ }^{5 \dagger} v_{\text {base }}-t_{\text {com } 1}-t_{\text {com } 2}-\left[t_{c o m} 3-h_{\text {top } 3}\right]-h_{\text {com } 4}\left(h_{\text {sp }}\right)$ & $\sim 2.0 \times 10^{-8}\left[\frac{a}{1.3 \times 10^{-2}}\right]$ & $\sim 2.0 \times 10^{-19}\left[\frac{a}{1.3 \times 10^{-2}}\right]^{\mathrm{m} / \sqrt{\mathrm{Hz}}}$ \\
\hline $6 t_{\text {tase }}-t_{\text {coml } 1}-t_{\text {com } 2}-t_{\text {com } 3}-\left[t_{\text {com } 4}-h_{s p}\right]$ & $\sim 3.4 \times 10^{-8}\left[\frac{z}{2.0 \times 10^{-3}}\right]^{\mathrm{m} / \mathrm{rad}}$ & $\sim 6.8 \times 10^{-19}\left[\frac{z}{2.0 \times 10^{-3}}\right]^{\mathrm{m} / \sqrt{\mathrm{Hz}}}$ \\
\hline $7 \quad h_{\text {base }}-t_{c o m 1}-t_{c o m 2}-t_{c o m} 3-\left[t_{c o m} 4-h_{s p}\right]$ & $\approx 2.3 \times 10^{-8}\left[\frac{z}{2.0 \times 10^{-3}}\right]$ & $\sim 2.3 \times 10^{-19}\left[\frac{z}{2.0 \times 10^{-3}}\right]^{\mathrm{m} /} / \sqrt{\mathrm{Hz}}$ \\
\hline
\end{tabular}

FIG. 27. The main transmission routes predicted to give rise to horizontal motion at the top of the four-stage stack. Horizontal, vertical, and tilting motions are denoted by $h, v$, and $t$, respectively. Round brackets indicate that the motion of the suspension point (sp) is equivalent to that of the center of mass (com) of the top plate of the stack. Square brackets are used where cross coupling of motions occurs within a metal plate. Here, $a$ is the vertical offset between the center of mass of a plate and the base level of the rubber supports for the stage above, and $z$ is the vertical offset between the pendulum suspension point and the center of mass of the top plate of the stack. We have used an offset $z$ of $2 \mathrm{~mm}$ as the reference offset since this would be a reasonable estimate of the accuracy to which the top plate center of mass and suspension point might be made coincident. The $\uparrow$ symbol in route 5 indicates that a $10 \%$ stiffness imbalance exists in the rubber springs of stage 1 .

the design stage, such effects are not expected to significantly alter the findings presented above. It should also be noted that this was not a full three-dimensional analysis. Rotations of the stack about a vertical axis were not accounted for in the finite element model: yet these could be important for the coupling of rotational ground noise to horizontal noise particularly if the suspension point of the pendulum was offset in the $y$ direction from the axis of symmetry of the stack (Fig. 1). Also stiffness imbalance in the rubber springs at either side of the stack in this situation could lead to more complicated transmission routes and would justify further analysis.

\section{ACKNOWLEDGMENTS}

We would like to thank J. E. Hall of Rutherford Appleton Laboratory and our colleagues in the Gravitational Waves Group at Glasgow University, in particular P. J. Veitch, for many useful discussions and suggestions. This work was supported by SERC and the University of Glasgow and one of us (C.A.C.) wishes to thank SERC for the award of a studentship.
${ }^{1}$ D. G. Blair, in The Detection of Gravitational Radiation, edited by D. G. Blair (Cambridge University, Cambridge, England, 1991), p. 73.

${ }^{2}$ J. Hough, B. J. Meers, G. P. Newton, N. A. Robertson, H. Ward, G. Leuchs, T. M. Niebauer, A. Rudiger, R. Schilling, L. Schnupp, H. Walther, W. Winkler, B. F. Schutz, J. Ehlers, P. Kafka, G. Schafer, M. W. Hamilton, I. Schutz, H. Welling, J. R. J. Bennett, I. F. Corbett, B. H. W. Edwards, R. J. S. Greenhalgh, and V. Kose, Proposal for a joint German-British interferometric gravitational wave detector [Tcchnical Report MPQ 147 (Max-Planck Institute of Technology and GWD/ 137/JH(89) (Rutherford Appleton Laboratory), September, 1989].

${ }^{3}$ The MacNeal-Schwendler Corporation, Handbook for Linear Analysis, MSC/Nastran Version 64 (California, 1985).

${ }^{4}$ The MacNeal-Schwendler Corporation, Handbook for Dynamic Analysis, MSC/Nastran Version 64 (California, 1983).

${ }^{5}$ R. Del Fabbro, A. Di Virgilio, A. Giazotto, H. Kautzky, V. Montelatici, and D. Passuello, Phys. Lett. A 133, 9 (1988).

${ }^{6} \mathrm{C}$. A. Cantley, PhD thesis, Glasgow University, Scotland, G12 8QQ, 1991.

7J. C. Snowdon, NBS Handbook 128, US Department of Commerce/ National Bureau of Standards (Washington, DC, 1979).

${ }^{8}$ R. J. S. Greenhalgh, Rutherford Appleton Laboratory, Chilton, Oxfordshire (private communication, 1989).

${ }^{9}$ FEGS Ltd. FAMresult Reference Manual (Cambridge, 1987).

${ }^{10} \mathrm{~J}$. E. Hall, Rutherford Appleton Laboratory, Chilton, Oxfordshire (private communication, 1991). 